\title{
Comparison of balloon dilation tract real-time monitored by ultrasound combined with endoscopic versus unitary ultrasound guided in percutaneous nephrolithotomy: a retrospective study
}

\section{Demao Ding}

the Second Affiliated Hospital of Anhui Medical University

\section{Daming Wang}

the Second Affiliated Hospital of Anhui Medical University

\section{Dongdong Xie}

the Second Affiliated Hospital of Anhui Medical University

Zhiqi Liu

the Second Affiliated Hospital of Anhui Medical University

Dengdian Wang

the Second Affiliated Hospital of Anhui Medical University

Enlai Li

the Second Affiliated Hospital of Anhui Medical University

Dexin Yu ( $\square$ yudx_urology@126.com )

the Second Affiliated Hospital of Anhui Medical University

\section{Research Article}

Keywords: Percutaneous nephrolithotomy, Balloon dilation, Ultrasound, Endoscopic

Posted Date: June 23rd, 2021

DOl: https://doi.org/10.21203/rs.3.rs-618405/v1

License: (c) (i) This work is licensed under a Creative Commons Attribution 4.0 International License. Read Full License 


\section{Abstract}

Background: The establishment of tract is very important in percutaneous nephrolithotomy. At present, balloon dilatation is widely used, but ultrasound or fluoroscopy guided balloon dilatation tract can not achieve real-time monitoring of the whole process, which easily leads to failure of tract establishment. we use ultrasound combined with endoscopic technology to realize the whole process monitoring of tract establishment. The purpose is to evaluate the safety and effectiveness of the technology.

Methods: Patients who underwent PCNL with only using the balloon dilator from January 2019 to April 2021 in the Second Affiliated Hospital of Anhui Medical University were retrospectively reviewed. They were divided into ultrasound combined with endoscopic guidance group and ultrasound-guided group. The demographic and renal stone characteristics, intraoperative and postoperative data were analyzed.

Results: A total of 72 patients were included in the study, including 35 patients in the ultrasound combined with endoscopic guidance group and 37 patients in the ultrasound-guided group. There was no difference in demographic and stone characteristics between the two groups. There were no significant differences between the two groups in surgical time, number of tract, hemoglobin decreased, stone free rate, ancillary treatment and postoperative complication rate. The tract establishment time of the ultrasound combined with endoscopic guidance group was significantly less than that of the ultrasoundguided group $(\mathrm{P}<0.05)$. There was no tract establishment failure in the ultrasound combined with endoscopic guidance group, while there were 7 cases in the ultrasound-guided group.

Conclusions: Ultrasound combined with endoscopic guidance can monitor the establishment of working tract of PCNL in real time. Compared with unitary ultrasonic guidance, the time of tract establishment can be shortened and the failure of tract establishment can be avoided.

\section{Background}

Percutaneous nephrolithotomy (PCNL) is the first choice for the treatment of renal calculi larger than $2 \mathrm{~cm}$ in diameter or complicated[1]. The location of puncture and the establishment of tract are the key points of successful operation. At present, fluoroscopy and ultrasound are the main methods of localization. The advantage of fluoroscopy positioning is that it can monitor the tract establishment, but the disadvantage is that it has radiation hazards and can not view adjacent organs around the kidney. The advantage of ultrasound localization is that it can view the organs around the kidney, puncture accurately and without radiation, but it is lack of monitoring in the process of tract establishment and requires higher technology to find residual stones[2, 3]. At present, the way of tract establishment is Amplatz dilation or balloon dilation. Pakmanesh et al studies have pointed out that although the cost of balloon dilator is higher, it is better than Amplatz dilation in safety and success rate[4]. Recent studies have pointed out that the whole process of establishing the tract with balloon dilator can be monitored by ultrasound, but for patients without hydronephrosis or obesity, there may still be not reached the desired calyx (short dilation) or tract displacement without fluoroscopy assistance, resulting in tract 
establishment failure or bleeding[5]. How to make the working tract of percutaneous nephrolithotomy safe and effective, we use ultrasound and endoscopic combined intrarenal surgery (ECIRS) technology to realize the whole process monitoring of tract establishment. The purpose is to discuss the safety and effectiveness of the technology.

\section{Materials And Methods}

Patients with renal calculi who underwent PCNL (balloon dilatation) in the Second Affiliated Hospital of Anhui Medical University from January 2019 to April 2021 were included in the analysis. They were divided into ultrasound combined with endoscopic guidance group and ultrasound-guided group. The study was approved by the hospital ethics committee and was followed up for at least 1 months. Demographic data included as age, gender and body mass index (BMI). Preoperative evaluation was performed by imaging and laboratory examination. All patients had an computed tomography (CT) scan and abdominal radiograph. Through the above radiological examination, stone size was determined by measuring the longest diameter; multiple stones is the sum of the length diameter of all stones. Stone surface area is calculated by formula (length $\times$ width $\times 3.14 \times 0.25$ )[6]. Guy's classification and STONE score were used to evaluate the complexity of renal calculi.

\section{Surgical Technique}

All patients were given general anesthesia. The patients in the ultrasound combined with endoscopic guidance group were in prone - split leg position, and then we performed retrograde operation and PCNL. The operation was performed by two urologists at the same time, one performed PCNL, the other performed transurethral retrograde surgery. First of all, lower the head side of the operating table to form an angle of $30^{\circ}$ with the horizontal line. The retrograde transurethral access to ureteroscopy (Karl Storz, Tuttlingen, Germany) was performed by surgeon 1 . Ureteroscopy was performed in the ureter and hydrophilic guide wire (Bard, Covington, GA) was placed. Ureteroscopy was withdrawn after entering the renal pelvis along the guide wire. Retrograde placement of flexible ureteroscope sheath(12/14F, Cook Urological) along the guide wire and flexible ureteroscope (Flex X-2, Karl Storz, Tuttlingen, Germany) was retrograde into renal pelvis. The distribution of calyces and calculi was examined by flexible ureteroscope, combined with ultrasound to determine the best puncture calyces. Under ultrasound guidance, surgeon 2 punctured the target calices with a $18 \mathrm{G}$ needle (Urovision, Germany) and the needle was visualized endoscopically entering the collecting system. Ultrasound combined with endoscopy guided and adjusted needle in the center of calyceal papilla "bull's-eye". After a 3.5F J-tip guide wire (Urovision, Germany) was inserted into renal pelvis under ultrasound and endoscopy guidance(Fig. 1A). 10F fascial dilator was advanced along the wire. The balloon dilator (Bard, USA) was placed along the guide wire under the continuous real-time guidance of ultrasound and endoscopy(Fig. 1B). After the tip of the dilator entered the calyces, the balloon was filled with water to make the pressure reach 25 ATM. The balloon dilator was completely expanded(Fig. 1C), and a 24F sheath was placed along the balloon(Fig. 1D). The sheath entered the calyces under endoscopic monitoring. After balloon removal, the 24F sheath was left as a working tract, and ultrasonic or pneumatic ballistic lithotripsy(Electro Medical Systems, Swiss 
LithoClast ${ }^{\circledR}$ Maste, Nyon, Switzerland) was used under the supervision of nephroscope. For stones that cannot be detected by nephroscope, flexible ureteroscope can move the stones to the field of nephroscope or holmium laser lithotripsy can be used directly. Finally, endoscopy combined with ultrasound was used to check whether there were residual stones. The ureteral stent and nephrostomy tube were placed and the operation was finished.

For the ultrasound-guided group, $5 \mathrm{~F}$ ureteral catheter was retrogradely inserted into the ureter to the renal pelvis under the lithotomic position, and then the position was changed to prone position. Under the guidance of ultrasound, the target calyces was determined and the puncture needle was used to enter the calyceal fornix. Under the real-time guidance of ultrasound, the guide wire was inserted into the calyces, and the balloon dilator (Bard, USA) was placed after the 10F fascial dilator was expanded in advance. Ultrasound showed that the tip of the dilator entered the calyces and began to inject water into the balloon. After the pressure reached 25 ATM and the balloon was completely expanded, 24F sheath was placed along the balloon to establish the working tract. Ultrasonic or pneumatic lithotripsy was used. After lithotripsy, ultrasound was used to check whether there were residual stones. Finally, ureteral stent and nephrostomy tube were placed.

The successful establishment of the tract is the first time that the distal end of the working sheath is accurately placed into the target calyx without damaging the renal pelvis and renal parenchyma. Too shallow (short dilation), too deep (ventral renal parenchyma injury) and secondary puncture were the failure of tract establishment. The time of channel establishment was from the beginning of puncture to the correct placement of the sheath in the target calyces. The operation time was calculated from the first retrograde access to urethral orifice to the placement of nephrostomy tube. CT was reexamined 1day and 4 weeks after operation to evaluate the effect of stone removal. Stone free status was defined as the stone was completely removed or the stone fragment was less than $2 \mathrm{~mm}$ [7]. Complications were graded according to the Clavien-Dindo system. To evaluate the safety and effectiveness of ultrasound combined with endoscopic guidance.

\section{Statistical analysis}

SPSS19.0 (SPSS Inc, USA) was used for statistical analysis. Continuous variables are expressed as mean $\pm S D$ and analyzed using the Student's $t$ test. Categorical variables are represented by frequency and percentage. The categorical data were compared by chi square test. $\mathrm{P}<0.05$ was considered to indicate statistical significance.

\section{Results}

A total of 72 patients were included in the study, including 35 patients in the ultrasound combined with endoscopic guidance group and 37 patients in the ultrasound-guided group. The demographic information and stone characteristics are presented in Table 1. There were no significant differences between the two groups in age, gender, BMI, history of kidney surgery, stone diameter, stone surface area, number of calyces involved by stone, STONE score and Guy's grade. 
All operations were successfully completed without major intraoperative complications. There were no significant differences between the two groups in surgical time, number of tract, hemoglobin $(\mathrm{Hb})$ decreased, stone free, ancillary treatment and postoperative complication rate (Table 1). The tract establishment time of the ultrasound combined with endoscopic guidance group was significantly less than that of the ultrasound-guided group $(P=0.003)$. There was no tract establishment failure in the ultrasound combined with endoscopic guidance group. Endoscopic monitoring showed that the "center of calyceal fornix" in ultrasound images was not completely the median of renal papilla (bull's-eye) (Fig. 2). 11 cases had the above problems and needed to adjust the needle angle. In the ultrasound-guided group, there were 7 cases of failed tract establishment, including 3 cases of successful search along the guide wire and 4 cases of puncture again.

\section{Discussion}

Balloon dilator is widely used in European and American countries to establish PCNL working tract[8]. Compared with fascia dilator, it can avoid step-by-step expansion and basically establish tract in one step, which can reduce the establishment time of tract. The continuous compression of injured vessels in the tract during balloon dilatation resulted in less blood loss[9]. Although balloon dilatation has many advantages in establishing tract, there are still some problems such as too shallow dilation (short dilation), too deep dilation and tract displacement in the process of dilatation, resulting in serious complications such as tract establishment failure, bleeding and renal parenchyma injury, especially in the patients without hydronephrosis[10,11] and obesity[12]. The correct puncture path and reasonable expansion method are effective methods to reduce the amount of blood loss[13]. At present, ultrasound and X-ray fluoroscopy methods can not achieve complete real-time monitoring in the whole process of balloon dilatation. In order to establish a more reasonable and safe tract, some researchers try different methods. The application of ultrasound combined with fluoroscopy can improve the success rate of tract establishment, but it can not fully monitor the whole process and ensure that the balloon dilator enters the calyces. X-ray examination is needed repeatedly, which is easy to increase radiation injury. Zhou et al study pointed out that ultrasound-guided puncture of the target calyces combined with optical puncture nephroscope (F4.8, Poly, Germany) to determine (the process of puncture can be visualized), and then placing balloon dilator for expansion can significantly improve the success rate of tract establishment[14]. However, the displacement of the kidney or the dilator may occur during the placement or expansion of the balloon dilator[4,5], which can not ensure that the balloon dilator accurately enters the calyces through the calyceal fornix for expansion.

The development of ECIRS technology makes the combination of antegrade and retrograde technology to provide a good treatment for complex renal calculi. In addition, it also provides a new idea for the establishment of tract. Grasso et al[15] first reported that percutaneous nephrolithotomy under the guidance of endoscopy combined with fluoroscopy was successfully performed in 7 patients. Kawahara et al[16] reported for the first time the retrograde nephrostomy assisted by flexible ureteroscope, in which the puncture needle was inserted from the middle calyces of kidney to the skin through flexible ureteroscope. However, due to the small space of calices and the poor puncture angle, retrograde 
puncture is difficult or failure probability is higher. In fact, the main purpose of all researchers is to improve the safety of tract establishment and reduce the fluoroscopy radiation time[17]. In order to further reduce the amount of radiation. Alsyouf et al[18] first proposed to establish the working tract of percutaneous nephrolithotomy under the guidance of endoscopy and ultrasound. The total fluoroscopy time was significantly reduced compared with the traditional operation method. Fluoroscopy was only used to confirm the sheath position and residual stones.

In our study, ultrasound combined with endoscopy guided balloon dilator was used to establish the working tract of percutaneous nephrolithotomy in prone-split leg position. There was no significant difference in operation time, number of tracts, decrease of hemoglobin, stone free rate and postoperative complications between the two groups, but the time of tract establishment was significantly shorter than that of ultrasound guidance alone. There were 7 cases failed to establish the tract for the first time in the ultrasound-guided group, but there was no failure in the ultrasound combined with endoscopy guided group. The combination of ultrasound and endoscopic guidance can determine the good puncture calibre, avoid short dilation and sheath displacement, shorten the time of tract establishment and prevent the failure of tract establishment. Endoscopic retrograde access to the renal pelvis can not only guide the establishment of the tract, but also assist the clearance of stones, avoid the establishment of additional tracts and improve the stone free rate[19]. The stone free rate of the ultrasound combined with endoscopy guided group was $91.4 \%$. The complication rate of ultrasound combined with endoscopy guided group was $22.9 \%$. There were no serious complications and massive hemorrhage requiring interventional embolization. As depicted in the Fig. 2, we found that in some cases, the so-called "calyceal fornix" under ultrasound images, after the puncture needle entered through the shortest path, it was actually the junction of the two calyces through flexible ureteroscopy monitoring. This may explain why there is still massive bleeding after ultrasound-guided puncture and expansion of "calyceal fornix". The monitoring under ureteroscope can guide the operator to adjust the angle of the puncture needle into the center of the renal papilla (bull's-eye), which can reduce the occurrence of bleeding[20].

Although the establishment of percutaneous nephroscope working tract under the guidance of endoscopy and ultrasound has great advantages, but it is not suitable for all patients. For the large stones in the renal pelvis and staghorn stones, the space in the renal pelvis and calyces is small, so it is impossible to realize endoscopic monitoring. In addition, the sample size of this study was small, and the risk factors of tract establishment failure or bleeding were not included.

\section{Conclusions}

In conclusion, the use of endoscope combined with ultrasound-guided balloon dilator to establish the working tract of percutaneous nephrolithotomy can shorten the time to establish the tract and improve the one-time success rate of tract establishment, and has certain advantages in stone free rate and prevention of complications.

\section{Abbreviations}


URSL: Ureteroscopy lithotripsy; PCNL: Percutaneous nephrolithotripsy; FURSL: Flexible ureteroscopy lithotripsy; ECIRS: Endoscopic combined intrarenal surgery; CT: Computed tomography;BMI: body mass index (BMl;

\section{Declarations}

Funding: Natural science research projects in Anhui Universities (KJ2020A0185)

Conflicts of interest/Competing interests: The authors declare that they have no conflict of interests.

Ethics approval and consent to participate: This study was carried out in accordance with the Helsinki Declaration and was approved by the Research Ethics Committee at Second Affiliated of Anhui Medical University. Written informed consent was obtained from all participants prior to their inclusion within this study.

Consent for publication: All of them read and approved the manuscript to publish.

Availability of data and material: The datasets used during this study available from the corresponding author on reasonable request.

Code availability: no applicable

Authors' contributions: all authors have read and approved the manuscript.

Demao DING and Daming Wang: Manuscript writing, Data collection and management; Dongdong Xie: Data analysis and management, Manuscript editing; Zhiqi Liu: Manuscript editing; Dengdian Wang Enlai Li: Manuscript editing; DX Yu: Project development, Manuscript editing, Protocol.

Acknowledgements: no applicable

\section{References}

1. Ghani KR, Andonian S, Bultitude M, et al. Percutaneous Nephrolithotomy: Update, Trends, and Future Directions. Eur Urol. 2016;70:382-396.

2. Ng FC, Yam WL, Lim T, et al. Ultrasound-guided percutaneous nephrolithotomy: Advantages and limitations. Investig Clin Urol. 2017;58:346-352.

3. Liu Q, Zhou L, Cai X, Jin T, Wang K. Fluoroscopy versus ultrasound for image guidance during percutaneous nephrolithotomy: a systematic review and meta-analysis. Urolithiasis. 2017;45:481-487.

4. Pakmanesh $\mathrm{H}$, Daneshpajooh A, Mirzaei M, et al. Amplatz versus Balloon for Tract Dilation in Ultrasonographically Guided Percutaneous Nephrolithotomy: A Randomized Clinical Trial. Biomed Res Int. 2019;2019:3428123. 
5. Wang S, Zhang Y, Zhang X, et al. Tract dilation monitored by ultrasound in percutaneous nephrolithotomy: feasible and safe. World J Urol. 2020;38:1569-1576.

6. Tiselius HG, Andersson A. Stone burden in an average Swedish population of stone formers requiring active stone removal: how can the stone size be estimated in the clinical routine? Eur Urol. 2003;43:275-281.

7. Zeng G, Zhong W, Pearle M, et al. European Association of Urology Section of Urolithiasis and International Alliance of Urolithiasis Joint Consensus on Percutaneous Nephrolithotomy. Eur Urol Focus. 2021.

8. Lopes T, Sangam K, Alken P, et al. The Clinical Research Office of the Endourological Society Percutaneous Nephrolithotomy Global Study: tract dilation comparisons in 5537 patients. J Endourol.2011;25:755-762.

9. Peng P, Lai S, Seery S, et al. Balloon versus Amplatz for tract dilation in fluoroscopically guided percutaneous nephrolithotomy: a systematic review and meta-analysis. BMJ Open. 2020;10:e35943.

10. Jin W, Song Y, Fei X. The Pros and cons of balloon dilation in totally ultrasound-guided percutaneous Nephrolithotomy. BMC Urol. 2020;20:82.

11. Usawachintachit M, Tzou DT, Hu W, et al. X-ray-free Ultrasound-guided Percutaneous Nephrolithotomy: How to Select the Right Patient? Urology. 2017;100:38-44.

12. Bayne DB, Usawachintachit M, Tzou D, et al. Increasing Body Mass Index Steepens the Learning Curve for Ultrasound-guided Percutaneous Nephrolithotomy. Urology. 2018;120:68-73.

13. Peng PX, Lai SC, Ding ZS, et al. One-shot dilation versus serial dilation technique for access in percutaneous nephrolithotomy: a systematic review and meta-analysis. BMJ Open. 2019;9:e25871.

14. Zhou M, He X, Zhang Y, et al. Optical puncture combined with balloon dilation PCNL vs. conventional puncture dilation PCNL for kidney stones without hydronephrosis: a retrospective study. BMC Urol. 2019;19:122.

15. Grasso M, Lang G, Taylor FC. Flexible ureteroscopically assisted percutaneous renal access. Tech Urol. 1995;1:39-43.

16. Kawahara $\mathrm{T}$, Ito $\mathrm{H}$, Terao $\mathrm{H}$, et al. Ureteroscopy assisted retrograde nephrostomy: a new technique for percutaneous nephrolithotomy (PCNL). BJU Int. 2012;110:588-590.

17. Isac W, Rizkala E, Liu X, Noble M, Monga M. Endoscopic-guided versus fluoroscopic-guided renal access for percutaneous nephrolithotomy: a comparative analysis. Urology. 2013;81:251-256. 
18. Alsyouf M, Arenas JL, Smith JC, et al. Direct Endoscopic Visualization Combined with Ultrasound Guided Access during Percutaneous Nephrolithotomy: A Feasibility Study and Comparison to a Conventional Cohort. J Urol. 2016;196:227-233.

19. Hamamoto S, Yasui T, Okada A, et al. Developments in the Technique of Endoscopic Combined Intrarenal Surgery in the Prone Split-leg Position. Urology. 2014;84:565-570.

20. Hamamoto S, Unno R, Taguchi K, et al. A New Navigation System of Renal Puncture for Endoscopic Combined Intrarenal Surgery: Real-time Virtual Sonography-guided Renal Access. Urology. 2017;109:44-50.

\section{Tables}

Table 1. Comparison of demographics and stone characteristics between the two groups

\begin{tabular}{|c|c|c|c|}
\hline Variables & observation group & control group & P value \\
\hline $\begin{array}{l}\text { No. of patients } \\
\text { Age (years) }\end{array}$ & $\begin{array}{l}35 \\
55.5 \pm 13.4\end{array}$ & $\begin{array}{l}37 \\
52.3 \pm 11.4\end{array}$ & 0.275 \\
\hline $\begin{array}{l}\text { Sex } \\
\quad \text { Male (\%) } \\
\text { Fmale (\%) }\end{array}$ & $\begin{array}{l}25(71.4) \\
10(28.6)\end{array}$ & $\begin{array}{l}26(70.3) \\
11(29.7)\end{array}$ & 0.914 \\
\hline BMI $\left(\mathrm{kg} / \mathrm{m}^{2}\right)$ & $25.1 \pm 3.6$ & $25.3 \pm 3.5$ & 0.806 \\
\hline $\begin{array}{l}\text { History of kidney surgery, n (\%) } \\
\text { PCNL }\end{array}$ & & & 0.866 \\
\hline $\begin{array}{l}\quad \text { Open nephrolithotomy } \\
\text { Renal stone size (mm) } \\
\text { Renal stone surface area }\left(\mathrm{mm}^{2}\right)\end{array}$ & $\begin{array}{l}3 \\
31.1 \pm 8.8 \\
669.5 \pm 234.5\end{array}$ & $\begin{array}{l}6 \\
30.8 \pm 10.3 \\
651.9 \pm 228.9\end{array}$ & $\begin{array}{l}0.920 \\
0.748\end{array}$ \\
\hline $\begin{array}{l}\text { Number of calyces involved by stone, } \mathrm{n}(\%) \\
<3 \\
\geq 3 \\
\text { STONE score }\end{array}$ & $\begin{array}{l}18(51.4) \\
17(48.6) \\
8.1 \pm 1.0\end{array}$ & $\begin{array}{l}16(43.2) \\
21(56.8) \\
8.3 \pm 0.9\end{array}$ & $\begin{array}{l}0.487 \\
0.410\end{array}$ \\
\hline $\begin{array}{l}\text { Guy's grade, n (\%) } \\
\begin{array}{c}\square-\square \\
\square-\square\end{array}\end{array}$ & $\begin{array}{l}18(51.4) \\
17(48.6) \\
\end{array}$ & $\begin{array}{l}18(48.6) \\
19(51.6)\end{array}$ & 0.814 \\
\hline
\end{tabular}

Table 2. Surgery Parameters and Postoperative Complications 


\begin{tabular}{llll}
\hline Variables & observation group & control group & P value \\
\hline Surgical time (min) & $80.7 \pm 29.9$ & $86.8 \pm 36.0$ & 0.438 \\
Number of tract, n (\%) & $32(91.4)$ & $30(81.1)$ & 0.353 \\
1 & $3(8.6)$ & $7(18.9)$ & 0.003 \\
$2-3$ & $7.6 \pm 2.0$ & $10.5 \pm 5.0$ & 0.370 \\
Tract establishment time (min) & 0 & $11.8 \pm 9.8$ & 0.124 \\
Tract establishment failed, n (\%) & $9.6 \pm 7.9$ & $29(78.4)$ & 0.181 \\
Hb drop (g/L) & $32(91.4)$ & $7(18.9)$ & \\
Stone free, n (\%) & $2(5.7)$ & 3 & 0.108 \\
Ancillary treatment, n (\%) & 0 & 3 & \\
Second PCNL & 1 & $22(59.5)$ & \\
SWL & 1 & $13(35.1)$ & \\
FURSL & & 3 & \\
Postoperative complications & $27(77.1)$ & 7 & \\
Clavien grade 0, n (\%) & $7(20.0)$ & 1 & \\
Clavien grade I, n (\%) & 2 & 1 & \\
Transient fever >38.5 ${ }^{\circ} \mathrm{C}$ & 3 & 1 & \\
Hemorrhage, non transfusion & 1 & 1 & \\
other & $1(2.9)$ & & \\
Clavien grade II, n (\%) & 1 & 0 & \\
Blood transfusion & 0 & & \\
Clavien grade $\geq \mathrm{III}, \mathrm{n}(\%)$ & 0 & & \\
Interventional embolization for hemostasis & & & \\
\hline
\end{tabular}

Figures 

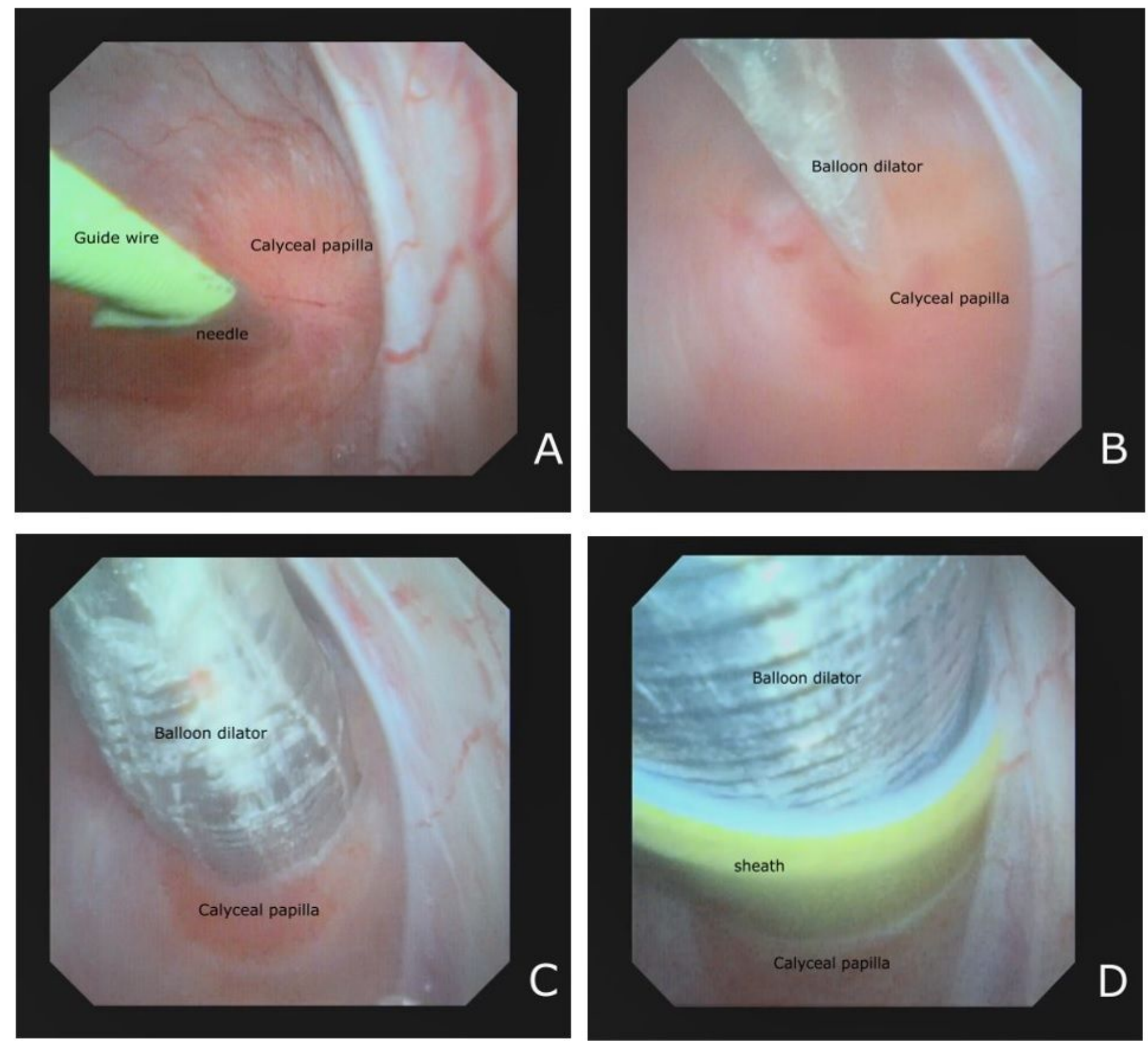

Figure 1

A: The puncture needle and guide wire pass through the center of calyceal papilla. B: The balloon dilator was placed along the guide wire under the monitoring of flexible ureteroscope. C: The balloon dilator dilated completely at the calyceal papilla. D: Sheath placement along the balloon under the monitoring of ureteroscope. 

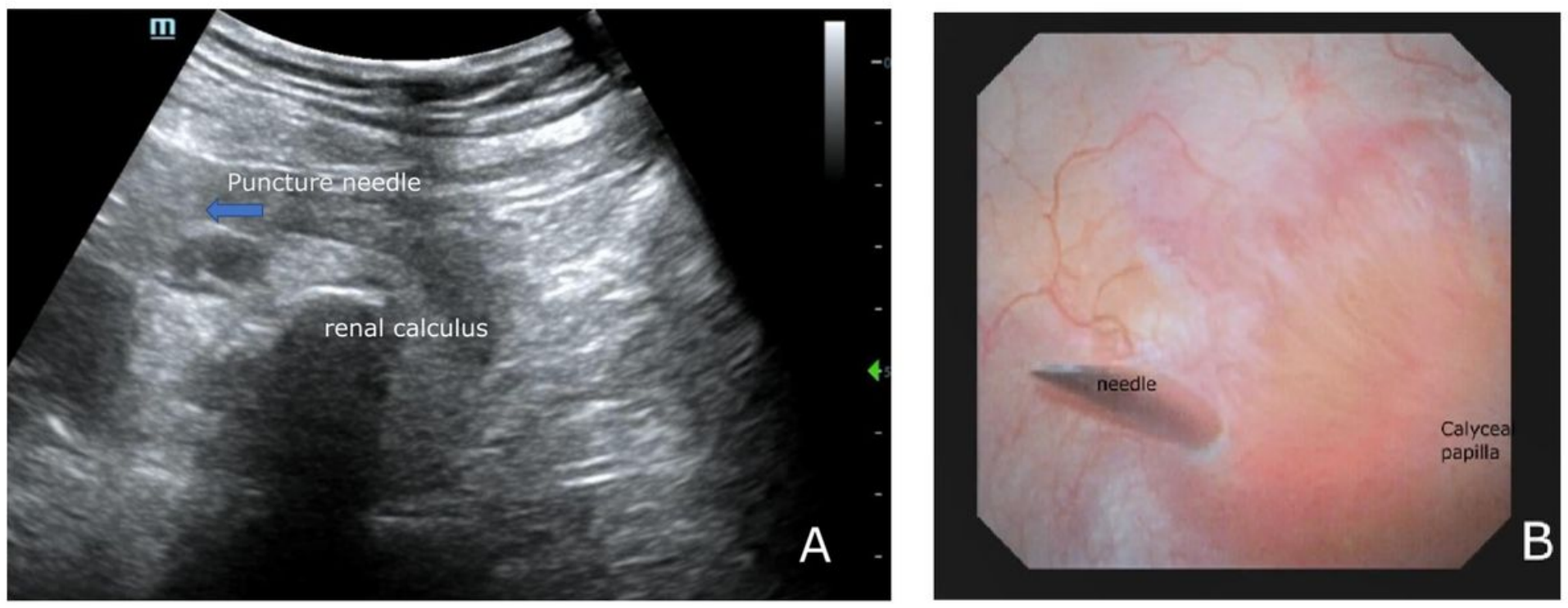

\section{Figure 2}

A: Ultrasound guided puncture needle in the "calyceal fornix" of ultrasound images into the calyces. B: Endoscopic monitoring showed that the puncture needle entered at the junction of the two calyces. 